\title{
NOTIZEN
}

\section{Darstellung von Gramin- $\left[{ }^{14} \mathrm{C}\right]$, $\beta$-Indolylessigsäure- $\left[{ }^{14} \mathrm{C}\right]$ und $d l$-Tryptophan- $\left[{ }^{14} \mathrm{C}\right]$ über Formaldehyd- $\left[{ }^{14} \mathrm{C}\right]$}

Darstellung von Aldehyden aus Carbonsäuren. V. Mitt.

Von Friedr. Weygand und Hanswerner Linden Chemisches Institut der Universität Tübingen

(Z. Naturforschg. 9 b, 682-683 [1954]; eingeg. am 20. Sept. 1954)

Kürzlich haben wir darüber berichtet ${ }^{1}$, daß $\mathrm{CO}_{2}$ unter definierten Bedingungen mit Hilfe von Lithiumaluminiumhydrid in Tetrahydrofuran in Ausbeuten bis zu 65\% zu Formaldehyd im mMol-Maßstab reduziert werden kann. Dadurch wird erstmals hochradioaktiver Formaldehyd leicht zugänglich.

Inzwischen haben wir Vorschriften für die Darstellung von Gramin-[14C] (= 3-Indolylmethyl-[14C]-dimethylamin), $\beta$-Indolylessigsäure-[2-14C] und $d, l$-Tryptophan[3-14 C] der Aktivität $1 \mathrm{mC} / \mathrm{mMol}$ ausgearbeitet, da diese Verbindungen für biochemische Versuche von großem Interesse sind und von verschiedenen Instituten benötigt wurden.

Es zeigte sich, daß das als Lösungsmittel verwendete Tetrahydrofuran beim Arbeiten im mMol-Maßstab die Mannich-Kondensation mit Indol und Dimethylamin unter den üblichen Reaktionsbedingungen ${ }^{2}$ stört. Das Tetrahydrofuran wurde daher im Hochvakuum (Vorlage mit flüssigem Stickstoff gekühlt) bei $0^{\circ}$ abgezogen, wobei die dazu benötigte Zeit in die Reduktionszeit ${ }^{1}$ einbezogen wurde. Der resultierende, den Formaldehyd enthaltende Trockenkomplex wurde sodann bei $-70^{\circ}$ mit etwas Methanol zerlegt, worauf die Kondensation ausführbar war. $\mathrm{Die} \mathrm{Li}$ - und $\mathrm{Al}-\mathrm{Salze}$ störten nicht. Auf das eingesetzte Bariumcarbonat ( $2 \mathrm{mMol}$ der Aktivität 1,05 $\mathrm{mC} / \mathrm{mMol}$ ) berechnet, erhielten wir Gramin in einer Ausbeute von $43-45 \%$ in mehreren Versuchen, $\beta$-Indolylessigsäure in einer Ausbeute von $75 \%$, ber. auf Gramin und $d, l$-Tryptophan in einer Ausbeute von 69\%, ber. auf Gramin (ohne Aufarbeitung der Mutterlaugen). Die Herstellung der genannten Verbindungen mit noch höherer Aktivität, z. B. $10 \mathrm{mC} / \mathrm{mMol}$, ist ohne weiteres möglich. Diese Aktivität könnte für pflanzenphysiologische Versuche mit $\beta$-Indolylessigsäure notwendig sein, da sie in äußerst geringen Konzentrationen ihre Wirkungen entfaltet.

Die gleichen Verbindungen, aber schwach radioaktiv markiert, wurden bereits von $\mathrm{Heidelberger} 3$ aus Formaldehyd, aus Methanol-14C durch Dehydrierung dargestellt, gewonnen.

$$
\text { Beschreibung der Versuche }
$$

3 - Indolylmethyl- $\left[{ }^{14} \mathrm{C}\right]-\mathrm{dimethylamin}$ (= Gramin). Aus 396,2 $\mathrm{mg} \mathrm{Ba}^{14} \mathrm{CO}_{3}$ (Aktivität $2 \mathrm{mC}$ )

$1 \mathrm{~F}$. Weygand u. H. Linden, Angew. Chem. 66, 174 [1954].

$2 \mathrm{H}$. K ühn u. O. S te in, Ber. dtsch. chem. Ges. 70, 567 [1937]; H. R. Snyder u. C. W. S mith, J. Amer. chem. Soc. 66, 200 [1944]. wurde mit Schwefelsäure in einer Hochvakuumapparatur das $\mathrm{CO}_{2}$ frei gemacht und mit Hilfe von flüssigem Stickstoff auf eine Lösung kondensiert, die $90 \mathrm{mg}$ Lithiumaluminiumhydrid (85-proz. nach der Menge an Wasserstoff, die durch Glykol in Tetrahydrofuran frei gemacht wird) in $9 \mathrm{ccm}$ abs. Tetrahydrofuran enthielt. Als Reaktionsgefäß diente ein Kölbchen von $50 \mathrm{ccm}$ Inhalt, das über ein abnehmbares Zwischenstück mit sehr weitem Hahn an die Hochvakuum-Apparatur angeschlossen war.

Nach dem Auftauen des Reaktionsgemisches an der Luft wurde das samt Verbindungsstück abgenommene Kölbchen in Eiswasser gebracht und genau 1/4 Stde. unter gelegentlichem Umschütteln darin gelassen. Aus dem wieder an die Hochvakuum-Apparatur gebrachten Kölbchen wurde das Tetrahydrofuran im Hochvakuum abdestilliert (Vorlage mit flüssigem Stickstoff gekühlt; das Kölbchen beschlägt sich mit einer Eisschicht). Dauer etwa 22 Minuten. Gesamte Reaktionsdauer also etwa 37 Min.

Nach dem Einlassen von Luft oder Stickstoff in die Apparatur wurde das Kölbchen abgenommen, auf $-80^{\circ}$ abgekühlt und vorsichtig mit $1,0 \mathrm{ccm}$ Methanol zur Zerlegung des Trockenkomplexes versetzt. Nun wurde eine bei $0^{\circ}$ bereitete Mischung von $1,2 \mathrm{ccm}$ wäßrigem Dimethylamin $(=428 \mathrm{mg}$ Dimethylamin $=9,5 \mathrm{mMol})$ in $5 \mathrm{ccm}$ Eisessig sowie $1 \mathrm{ccm}$ Wasser zugesetzt. Das verschlossene Gefäß wurde 1 Min. geschüttelt. Nach der Zugabe von $234 \mathrm{mg}$ Indol $(2 \mathrm{mMol})$ wurde $20 \mathrm{Stdn}$. lang geschüttelt oder stehen gelassen unter gelegentlichem Umschütteln. Nun wurde unter Kühlung mit Eis mit $4 \mathrm{~g}$ Natriumhydroxyd in $40 \mathrm{ccm}$ Wasser alkalisch gemacht und über Nacht in den Eisschrank gesetzt. Die ausgeschiedene krümelige Masse wurde durch die Fritte eines Extraktionsrohres filtriert und 3-mal mit je 10-15 ccm Wasser gewaschen (die Filtrate, die Methanol-14 $\mathrm{C}$ und Natriumformiat-14C enthalten, können auf diese Verbindungen hin aufgearbeitet werden).

Die organischen Verbindungen wurden sodann mit $40 \mathrm{ccm}$ Tetrahydrofuran extrahiert. Nach dem Abdestillieren des Lösungsmittels wurde der braune Rückstand in $50 \mathrm{ccm}$ Petroläther $\left(60-90^{\circ}\right)$ durch Erhitzen aufgenommen. Es hinterblieb eine braune Schmiere, die hochradioaktiv war und vermutlich $\mathrm{Di}$ - $(\beta$-indolyl)-methan$\left[{ }^{14} \mathrm{C}\right]$ enthält. Aus dem Petroläther kristallisierten nach dem Einengen auf etwa $30 \mathrm{ccm}$ und stehenlassen im Eisschrank über Nacht zunächst $117 \mathrm{mg}$ Gramin-[14C] aus. Nach dem Einengen der Mutterlaugen auf $3 \mathrm{ccm}$ erhielt man noch $38 \mathrm{mg}$; Schmp. $129^{\circ}$ bzw. $124-126^{\circ}$ (Mikroschmp.). Gesamtausbeute $155 \mathrm{mg}=44,6 \%$, ber. auf das eingesetzte $\mathrm{Ba}^{14} \mathrm{CO}_{3}$.

$\beta$-Indolylessigsäure- $\left[2-{ }^{14} \mathrm{C}\right]$. Die Darstellung aus Gramin erfolgte nach einer etwas abgeänderten Vorschrift von Snyder und Pilgrim ${ }^{4} .128 \mathrm{mg}$ Gramin- $\left[{ }^{14} \mathrm{C}\right](0,74 \mathrm{mMol})$ und $181 \mathrm{mg}(3,7 \mathrm{mMol}) \mathrm{Na}-$

${ }_{3}$ C. He id el berger, J. biol. Chemistry 179, 139 [1949].

${ }_{4}^{4}$ H. R. S n y de r u. F. J. P il g r i m, J. Amer. chem. Soc. 70, 3770 [1948]. 
triumcyanid wurden mit $2 \mathrm{ccm}$ Äthanol und $0,5 \mathrm{ccm}$ Wasser 95 Stdn. lang unter magnet. Rühren unter Rückfluß erhitzt. Der Alkohol wurde abgedampft, der Rückstand mit $0,1 \mathrm{~g} \mathrm{NaOH}$ in $0,5 \mathrm{ccm}$ Wasser versetzt, worauf noch $5 \mathrm{Stdn}$. unter Rückfluß gekocht wurde. Unter Eiskühlung wurde sodann mit $1 \mathrm{ccm}$ konz. Salzsäure vorsichtig versetzt, wobei ein Niederschlag ausfiel, der abgesaugt wurde. Der beim Eindampfen der Mutterlauge hinterbleibende feste Rückstand wurde mit dem abgesaugten Niederschlag zusammen bei $70^{\circ}$ getrocknet und mit Dichloräthylen siedend aus einem Extraktionsrohr mit Glasfritte extrahiert. Die Lösung wurde auf etwa $10 \mathrm{ccm}$ eingeengt und über Nacht im Eisschrank stehen gelassen. Nach dem Absaugen und Waschen mit etwas Dichloräthylen lagen $98 \mathrm{mg}(\mathbf{7 5 , 4 \%}$ d. Th.) $\beta$-Indolylessigsäure-[2-14C] vor. Mikroschmp. $167-168^{\circ}$. In der Mutterlauge ist noch $\beta$-Indolylessigsäure enthalten, auf deren Gewinnung zunächst verzichtet wurde.

Skatyl-[methylen $\left.-{ }^{14} \mathrm{C}\right]$ - formaminomalon säure-diäthylester: $155 \mathrm{mg}$ Gramin-[14C], $272 \mathrm{mg}$ Formaminomalonsäure-diäthylester 5 und etwa $10 \mathrm{mg}$ gepulvertes Natriumhydroxyd wurden in 2,5 ccm abs. Xylol unter Einleiten von Stickstoff 7 Stdn. unter Rückfluß erhitzt. Die im Eisschrank über Nacht auskristallisierte Verbindung wurde abgesaugt und mit Petroläther gewaschen. Ausbeute $284 \mathrm{mg}(97,5 \%$ d. Th.), Mikroschmp. 167-169 ${ }^{\circ}$. Nach dem Umkristallisieren aus $2 \mathrm{ccm}$ Xylol, Waschen mit Petroläther und Trocknen bei $60^{\circ}$ im Vakuum über Paraffin lagen noch $264 \mathrm{mg}$ (90,5\% d. Th.), Mikroschmp. $177^{\circ}$, vor.

$d, l-\mathrm{T}$ ry p t o ph a n $-\left[3-{ }^{14} \mathrm{C}\right] .256 \mathrm{mg}$ Skatyl-[methylen $-{ }^{14} \mathrm{C}$ ] - formaminomalonsäure - diäthylester wurden mit 1,6 ccm Natronlauge (aus 2,3 g Natriumhydroxyd und $22 \mathrm{ccm}$ Wasser bereitet) $6 \mathrm{Stdn}$. unter Rückfluß im Ölbad $\left(120-130^{\circ}\right)$ erhitzt, nach Zugabe von $0,3 \mathrm{ccm}$ Eisessig wurde noch 2 Stdn. bei $130^{\circ}$ gehalten. Nach dem Stehen im Eisschrank saugte man das Tryptophan ab und wusch es mit wenig eiskaltem Wasser. Es wurde aus wenig Eisessig unter Zugabe von Benzol (zur klar filtrierten Lösung) umkristallisiert und in der Pistole getrocknet. Ausb. 119 mg. Mikroschmp. 235 (Zers.). Weitere Umkristallisation aus sied. Wasser + Alkohol.

5 H. H e $1 \mathrm{l}$ m a n n, Hoppe-Seyler's Z. physiol. Chem. 284, 163 [1949]. Herrn Dr. H ell m a n n danken wir bestens für die Überlassung dieser Verbindung.

\section{Notiz zur Stereochemie des Meteloidins}

Von Alex Heusner

Wissenschaftliche Abteilung

der Firma C. H. Boehringer Sohn, Ingelheim a. Rh. (Z. Naturforschg. 9 b, 683-684 [1954]; eingegangen am 9. Aug. 1954)

Der bis vor kurzem noch unbekannte räumliche Bau des Alkaloids Meteloidin (3.6.7-Trioxy-tropan-3-tiglinsäureester) ist durch eine soeben erschienene Arbeit von F od or und Mitarbb. 1 in den wichtigsten Punkten aufgeklärt worden. Die ungarischen Autoren haben aus Teloidin, dem Verseifungsprodukt des Alkaloids, ein

1 G. Fodor, J. Tóth u. I. V incze, Helv. chim. Acta 37, 907 [1954]. quartäres Lactonjodid dargestellt, dem sie Formel (I) zuschreiben, womit für die cis-Glykolgruppierung des Fünfringes $\beta$-Stellung (cis zur Stickstoffbrücke) nachgewiesen ist ${ }^{2}$.

Die angeführten experimentellen Belege sichern die Konstitution des Lactons im Sinne von (I) weitgehend. Es erscheint jedoch, worauf auch F od or und Mitarbb. ${ }^{1}$ hinweisen, wünschenswert, noch die $\alpha$-Stellung der 3Oxygruppe (trans zur Stickstoffbrücke) zu beweisen, um mit absoluter Sicherheit auszuschließen, daß der Lactonringschluß etwa unter Einbeziehung dieser Hydroxylgruppe erfolgt ist.

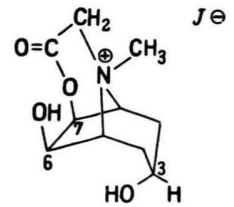

(I)

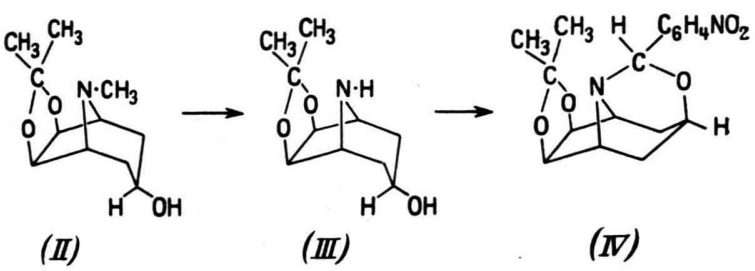

Wir haben in der letzten Zeit gleichfalls Versuche zur Stereochemie des Meteloidins angestellt, von denen im Folgenden diejenigen aufgeführt seien, die die noch fragliche Stellung der 3-Oxygruppe zum Gegenstand hatten.

Bei diesen Versuchen wurde Pseudoteloidin-acetonid (II) 3, 4 durch alkalische Permanganat-Oxydation seiner 3-Acetylverbindung 3 zu Nor-pseudoteloidin-acetonid (III) entmethyliert (aus Benzol lange farblose Nadeln vom Schmp. $177,5-179,5^{\circ}$ ). Umsetzung von (III) mit $p$-Nitrobenzaldehyd in Chlorbenzol 5 ergab in über 90 -proz. Ausbeute das 2-( $p$-Nitrophenyl)-tetrahydrooxazin des Norpseudoteloidin-acetonids (IV) (aus Isopropyläther farblose Blöcke vom Schmp. $134-135^{\circ}$ ). Aus dieser Reaktion, die für Pseudoteloidin cis-Stellung von 3-Oxygruppe und Stickstoffbrücke beweist, folgt zwangsläufig für seine 3epimere Verbindung Teloidin trans-Stellung dieser Gruppen. Damit ist einerseits gezeigt, daß Teloidin der gleichen sterischen Reihe $(3 \alpha)$ wie Tropin angehört, und andererseits erfahren die Versuche von F od o r und Mitarbb. ${ }^{1}$ über die räumliche Anordnung der Substituenten im Fünfring ihre letzte Ergänzung. Die Konfiguration von Meletoidin als Ester des $3 \alpha, 6 \beta, 7 \beta$-Trioxytropans (vgl. I) erscheint somit gesichert.

2 Cis-Stellung dieser beiden Hydroxylgruppen untereinander hatte sich bereits aus der Totalsynthese ergeben: C. Schöpf u. W. Arnold, Liebigs Ann. Chem. 558, 109 [1947].

3 J. C. She ehan u. E. R. B is s e 11, J. org. Chemistry 19, 270 [1954].

4 A. H e u s ner, Chem. Ber. 87, 1032 [1954]

5 Dieses Verfahren ist in der Tropanreihe erstmals von E. H a r d e g ge r u. H. Ot t (Helv. chim. Acta 36, 1186 [1953]) zur Aufklärung der Konfiguration des Pseudotropins angewandt worden. 